\title{
Novel multiple access for cooperative networks with nakagami-m fading: system model and performance analysis
}

\author{
Tu-Trinh Thi Nguyen ${ }^{1}$, Dinh-Thuan Do ${ }^{2}$ \\ ${ }^{1}$ Faculty of Electronics Technology, Industrial University of Ho Chi Minh City, Vietnam \\ ${ }^{2}$ Wireless Communications Research Group, Ton Duc Thang University, Vietnam \\ ${ }^{2}$ Faculty of Electrical \& Electronics Engineering, Ton Duc Thang University, Vietnam
}

\begin{tabular}{l} 
Article Info \\
\hline Article history: \\
Received Apr 18, 201 \\
Revised Aug 13, 20 \\
Accepted Jan 15, 2020 \\
\hline Keywords: \\
NOMA \\
Outage probability \\
Throughput
\end{tabular}

Throughput

\begin{abstract}
This study considers the performances of a downlink non-orthogonal multiple access (NOMA) network over Nakagami-m fading channels. This scheme employs two hop transmission. We first examine the analytical outage probability expression for each user and then these results are verified by Monte Carlo simulations. Based upon the outage probability, the maximal throughput are obtained and analyzed under different parameters. Furthermore, the effects of Nakagami-m channel fading on the probability and throughput are investigated. The results indicate that the performance of the NOMA system is excellent as appropriate selection of simulated parameters. This analysis can be recommended for designing the NOMA systems in the future fifth-generation wireless communication.
\end{abstract}

Copyright $@ 2020$ Institute of Advanced Engineering and Science. All rights reserved.

Corresponding Author:

Dinh-Thuan Do,

Faculty of Electrical \& Electronics Engineering, Ton Duc Thang University,

Ho Chi Minh City, Vietnam.

Email: dodinhthuan@tdtu.edu.vn

\section{INTRODUCTION}

As one of the bright networks, the non-orthogonal multiple access (NOMA) technique has been proposed to improved spectral efficiency for the fifth generation $(5 \mathrm{G})$ wireless communication system $[1,2]$. Unlike the conventional orthogonal multiple access (MA) technique, NOMA can divide the power domain to multiple users to access to networks [3]. At the base station (BS) side, superposition coding is employed to support the signals of multiple users multiplexed before transmitting. At the users' side, the inter-user interference can be eliminated by employing successive interference cancellation (SIC). In addition to power domain based multiplexing, an another distinctive feature of NOMA is user fairness [4]. In these NOMA schemes, more power is allocated to users who possess worse channel conditions while users with higher channel gains require less power. As a result, NOMA communication system can be able realized to improve trade-off between user fairness and the performance. By providing significant advantages over the conventional orthogonal multiple access protocols, several studies have shown promising deployment of NOMA to achieve higher throughput and faster traffic. Recently, energy harvesting and relaying scheme are proposed to provide extended coverage and improved outage performance in many scenarios such as [5-16].

Full-duplex scheme is proposed to provide bandwidth efficiency for such relaying networks [5, 6]. Using relaying scheme, the relay assists to far users achieve more chances to remain their operations. For example, these systems can be able to eliminate interference such as co-channel interference (CCI) [8]. This paper first examine relaying network together with employing PDMA under impact of CCI on its performance analysis. In order to further enhance the reliability of NOMA, this technique has been extended to cooperative transmission scenarios [5-8]. In cooperative NOMA (C-NOMA) networks, users and relays cooperate to combat channel fading and improve the performances. 
Furthermore, improved system performance as combining relaying networks with NOMA as in [17-24]. The authors in [17] considered relay selection to improve performance of far users in such NOMA. Interestingly, secure performance can be satisfied in NOMA based on relay selection [17]. Wireless powered networks are employed to support NOMA prolong life-time as power constraint-assisted device [18, 19]. The authors in [18] investigated imperfection in channel state information (CSI) on system performance of C-NOMA. However, these systems are considered performance in term of Rayleigh fading channel. The bad situation of imperfect CSI can be shown in degradation performance as in $[19,21]$. To consider Nakagami-m fading, Men et al. [24] have investigated of the C-NOMA system with respect to the outage probability, but the diversity technique was not considered. Actually, the performance is further enhanced by diversity reception. Unfortunately, the closed-forms of the performance metrics are hard to derive in such a case. Thus, it is very challenging to provide an accurate analysis of the C-NOMA system with relaying scheme and Nakagami-m fading. Motivated by this requirement, we consider a relaying transmission for downlink in NOMA network with respect to Nakagami-m fading in this study.

\section{SYSTEM MODEL AND OUTAGE PERFORMANCE ANALYSIS}

\subsection{System model}

Consider a downlink for this NOMA network in this system model. This system model consists of one single-antenna base station (BS), one single-antenna relay (R) and two single-antenna users $\left(D_{1}, D_{2}\right)$, as shown in Figure 1. In this scenario, the BS serves the two users in the same time. Two far NOMA users can be activated thanks to the help of a decode-and-forward (DF) relay. It is assumed that the channel gain of the BS-relay link experiences Nakagami-m distribution with fading parameters $\mathrm{m}$. Each of the channel gains is assumed to be independent.

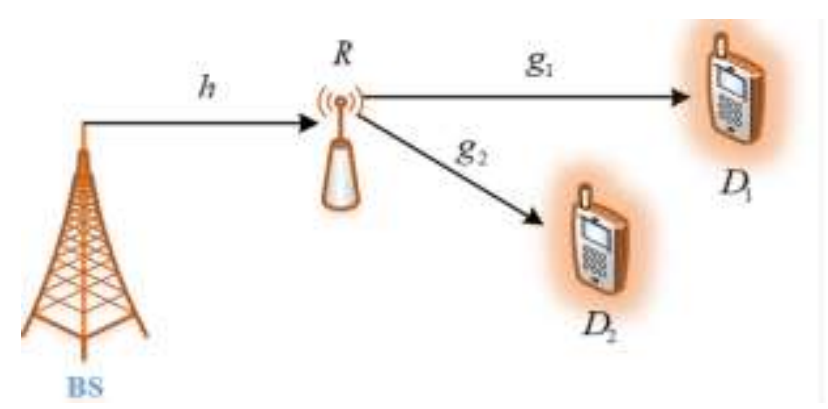

Figure 1. System model of NOMA over Nakagami-m fading channel

Based on the aforementioned assumptions, the received signals at $D_{k}, k=1,2$ can be written as follows:

$$
y_{k}^{d}=\sqrt{P} g_{k} \sum_{i=1}^{2} \sqrt{a_{i}} x_{i}+w_{k}^{d}
$$

where $g_{k}$ is channel coefficient from relay to $D_{k}, \mathrm{P}$ denotes the transmission power at relay and $w_{k}^{d}$ denotes the AWGN at $D_{k}$, i.e., $w_{k}^{d} \sim \mathcal{C N}\left(0, \sigma^{2}\right), x_{1}, x_{2}$ are the normalized signal for $D_{1}, D_{2}$, respectively. It is assumed that $E\left\{x_{1}^{2}\right\}=E\left\{x_{2}^{2}\right\}=1, a_{1}, a_{2}$ are power allocation factors. To stipulate better fairness between the users, we assume that $a_{1}>a_{2}$ satisfying $a_{1}+a_{2}=1$. The SINR at destination 1 in order to decode its own data can be written by:

$$
\gamma_{d_{1}}=\frac{a_{1} \rho_{r}\left|g_{1}\right|^{2}}{a_{2} \rho_{r}\left|g_{1}\right|^{2}+1}, .
$$


Additionally, user 2 only recovers its data after successfully decomposing user 1 data and applying SIC. Then, the SINR and SNR at second user $D_{2}$ to detect the first user symbol for SIC purpose and to discover its own data are respectively given by:

$$
\gamma_{d_{2} \leftarrow 1}=\frac{a_{1} \rho_{r}\left|g_{2}\right|^{2}}{a_{2} \rho_{r}\left|g_{2}\right|^{2}+1},
$$

We denote $\rho_{r}=\frac{P}{\sigma^{2}}$ as signal to noise ratio (SNR) at relay. Then the received SINR at $D_{2}$ to detect its own information is given by:

$$
\gamma_{d_{2}}^{p S I C}=a_{2} \rho_{r}\left|g_{2}\right|^{2}
$$

and,

$$
\gamma_{d_{2}}^{i p S I C}=\frac{a_{2} \rho_{r}\left|g_{2}\right|^{2}}{v \rho_{r}\left|k_{d_{1}}\right|^{2}+1} .
$$

\section{PERFORMANCE ANALYSIS}

We first call channel assumption: Assuming, all channel gain undergo independence Nakagami-m fading distribution. The PDF and CDF are given by

$$
\begin{aligned}
& f_{|z|^{2}}(x)=\frac{x^{m_{z}-1}}{\Gamma\left(m_{z}\right) \beta_{z}^{m_{z}}} \exp \left(-\frac{x}{\beta_{z}}\right), \\
& F_{|z|^{2}}(x)=1-\frac{1}{\Gamma\left(m_{z}\right)} \Gamma\left(m_{z}, \frac{x}{\beta_{z}}\right)=1-\exp \left(-\frac{x}{\beta_{z}}\right) \sum_{n=0}^{m_{z}-1} \frac{x^{n}}{n ! \beta_{z}^{n}},
\end{aligned}
$$

where $\beta_{z}=\lambda_{z} / m_{z}$ with $\lambda_{z}$ and $m_{z}$ represent the mean and integer fading factor, $m=m_{h}=m_{g_{1}}=m_{2}=m_{k_{1}}$, $\lambda_{h}=d_{1 r}^{\alpha}, \lambda_{g_{1}}=d_{r 1}^{-\alpha}, \lambda_{g_{2}}=d_{r 2}^{-\alpha}, \lambda_{k_{1}}=\kappa \lambda_{h}, d_{1 r}, d_{r 1}, d_{r 2}$ are distances related to these nodes, $\alpha$ is path-loss exponent.

\subsection{The outage probability for detecting ${ }^{x_{1}}$ at ${ }^{D_{1}}$}

We first consider the outage probability for detecting $x_{1}$ at $D_{1}$ can be expressed as:

$$
O P_{1}=1-\operatorname{Pr}\left(\gamma_{d_{1}} \geq \delta_{1}^{\text {th }}\right)
$$

where $\delta_{1}^{t h}=2^{2 R_{1}}-1$. After simple manipulations, we have following equation as

$$
\begin{aligned}
O P_{1} & =1-\operatorname{Pr}\left(\frac{a_{1} \rho_{r}\left|g_{1}\right|^{2}}{a_{2} \rho_{r}\left|g_{1}\right|^{2}+1} \geq \delta_{1}^{\text {th }}\right) \\
& =F_{\left|g_{1}\right|^{2}}\left(\frac{\delta_{1}^{\text {th }}}{a_{1} \rho_{r}-\delta_{1}^{\text {th }} a_{2} \rho_{r}}\right)
\end{aligned}
$$


3.2. The outage probability for detecting $x_{2}$ at $D_{2}$

We call $\delta_{2}^{t h}=2^{2 R_{2}}-1$ as threshold SNR. Next, we continue to determine outage performance of $D_{2}$ to detect $x_{2}$ and it is defined by:

$$
O P_{2}^{p S I C}=1-\operatorname{Pr}\left(\gamma_{d_{2} \leftarrow 1} \geq \delta_{1}^{t h}, \gamma_{d_{2}}^{p S I C} \geq \delta_{2}^{t h}\right)
$$

Plugging above values of (3) and (4) into (11), we have:

$$
\begin{aligned}
O P_{2}^{p S I C} & =1-\operatorname{Pr}\left(\frac{a_{1} \rho_{r}\left|g_{2}\right|^{2}}{a_{2} \rho_{r}\left|g_{2}\right|^{2}+1} \geq \delta_{1}^{\text {th }}, a_{2} \rho_{r}\left|g_{2}\right|^{2} \geq \delta_{2}^{t h}\right) \\
& =F_{\left|g_{2}\right|^{2}}\left(\mathcal{Q}_{1}\right)
\end{aligned}
$$

After the implementation of the calculation, we have the outage probability for detecting $x_{2}$ at $D_{2}$ can be formulated in closed-form as:

$$
\begin{gathered}
\qquad P_{2}^{p S I C}=1-\exp \left(-\frac{\mathcal{Q}_{1}}{\beta_{g_{2}}}\right) \sum_{n_{1}=0}^{m_{g_{2}}-1} \frac{\mathcal{Q}_{1}^{n_{1}}}{n_{1} ! \beta_{g_{2}}^{n_{1}}} \\
\mathcal{Q}_{1}=\max \left(\frac{\delta_{1}^{t h}}{a_{1} \rho_{r}-\delta_{1}^{t h} a_{2} \rho_{r}}, \frac{\delta_{2}^{t h}}{a_{2} \rho_{r}}\right)
\end{gathered}
$$

\subsection{The outage probability for detecting $x_{2}$ at $D_{2}$ with imperfect SIC}

The outage probability for detecting $x_{2}$ at $D_{2}$ in special case of imperfect SIC

$$
O P_{2}^{i p S I C}=1-\operatorname{Pr}\left(\gamma_{d_{2} \leftarrow 1} \geq \delta_{1}^{t h}, \gamma_{d_{2}}^{i p S I C} \geq \delta_{2}^{t h}\right)
$$

Plugging above values of (3) and (5) into (14), we have

$$
\begin{aligned}
& O P_{2}^{i p S I C}=1-\operatorname{Pr}\left(\frac{a_{1} \rho_{r}\left|g_{2}\right|^{2}}{a_{2} \rho_{r}\left|g_{2}\right|^{2}+1} \geq \delta_{1}^{t h}, \frac{a_{2} \rho_{r}\left|g_{2}\right|^{2}}{v \rho_{r}\left|k_{d_{1}}\right|^{2}+1} \geq \delta_{2}^{t h}\right) \\
& =1-\underbrace{\operatorname{Pr}\left(\left|g_{2}\right|^{2} \geq \frac{\delta_{1}^{t h}}{a_{1} \rho_{r}-a_{2} \rho_{r} \delta_{1}^{t h}},\left|k_{d_{1}}\right|^{2}<\max \left(0, \frac{1}{v \rho_{r}}\left(\frac{a_{2} \delta_{1}^{t h}}{\left(a_{1}-a_{2} \delta_{1}^{t h}\right) \delta_{2}^{t h}}-1\right)\right)\right)}_{\triangleq \triangleq} \\
& \underbrace{-\operatorname{Pr}\left(\left|g_{2}\right|^{2} \geq \frac{v \delta_{2}^{t h}}{a_{2}}\left|k_{d_{1}}\right|^{2}+\frac{\delta_{2}^{t h}}{a_{2} \rho_{r}},\left|k_{d_{1}}\right|^{2}>\max \left(0, \frac{1}{v \rho_{r}}\left(\frac{a_{2} \delta_{1}^{t h}}{\left(a_{1}-a_{2} \delta_{1}^{t h}\right) \delta_{2}^{t h}}-1\right)\right)\right)}_{\underline{\triangleq} \varphi_{2}}
\end{aligned}
$$

From (15), we can calculate $\varphi_{1}$ and $\varphi_{2}$ as follow: 


$$
\begin{aligned}
& \varphi_{1}=\operatorname{Pr}\left(\left|g_{2}\right|^{2} \geq \frac{\delta_{1}^{t h}}{a_{1} \rho_{r}-a_{2} \rho_{r} \delta_{1}^{t h}},\left|k_{d_{1}}\right|^{2}<\max \left(0, \frac{1}{v \rho_{r}}\left(\frac{a_{2} \delta_{1}^{\text {th }}}{\left(a_{1}-a_{2} \delta_{1}^{t h}\right) \delta_{2}^{t h}}-1\right)\right)\right) \\
& =\left(1-F_{\left|g_{2}\right|^{2}}\left(\frac{\delta_{1}^{t h}}{a_{1} \rho_{r}-a_{2} \rho_{r} \delta_{1}^{t h}}\right)\right) F_{\left|k_{d_{1}}\right|^{2}}(\underbrace{\max \left(0, \frac{1}{v \rho_{r}}\left(\frac{a_{2} \delta_{1}^{t h}}{\left(a_{1}-a_{2} \delta_{1}^{t h}\right) \delta_{2}^{t h}}-1\right)\right)}_{\cong \mu}) \\
& =\exp \left(-\frac{\delta_{1}^{t h}}{\left(a_{1} \rho_{r}-a_{2} \rho_{r} \delta_{1}^{t h}\right) \beta_{g_{2}}}\right)_{n=0}^{m_{g_{2}}-1} \frac{1}{n ! \beta_{g_{2}}^{n}}\left(\frac{\delta_{1}^{t h}}{a_{1} \rho_{r}-a_{2} \rho_{r} \delta_{1}^{t h}}\right)^{n} \\
& -\exp \left(-\frac{\delta_{1}^{t h}}{\left(a_{1} \rho_{r}-a_{2} \rho_{r} \delta_{1}^{t h}\right) \beta_{g_{2}}}-\frac{\mu}{\beta_{k_{d_{1}}}} \sum_{n=0}^{m_{g_{2}}-1} \sum_{n_{1}=0}^{m_{k_{d_{1}}}-1} \frac{1}{n ! \beta_{g_{2}}^{n}}\left(\frac{\delta_{1}^{t h}}{a_{1} \rho_{r}-a_{2} \rho_{r} \delta_{1}^{t h}}\right)^{n} \frac{\mu^{n_{1}}}{n_{1} ! \beta_{k_{d_{1}}}^{n_{1}}}\right.
\end{aligned}
$$

and,

$$
\begin{aligned}
\varphi_{2} & =\operatorname{Pr}\left(\left|g_{2}\right|^{2} \geq \frac{v \delta_{2}^{t h}}{a_{2}}\left|k_{d_{1}}\right|^{2}+\frac{\delta_{2}^{t h}}{a_{2} \rho_{r}},\left|k_{d_{1}}\right|^{2}>\max \left(0, \frac{1}{v \rho_{r}}\left(\frac{a_{2} \delta_{1}^{t h}}{\left(a_{1}-a_{2} \delta_{1}^{t h}\right) \delta_{2}^{t h}}-1\right)\right)\right) \\
& =\exp \left(-\frac{\delta_{2}^{t h}}{a_{2} \rho_{r} \beta_{g_{2}}}\right)^{m_{g_{2}}-1} \sum_{n_{2}=0}^{n_{2}} \sum_{n_{3}=0}^{n_{2}}\left(\begin{array}{l}
n_{2} \\
n_{3}
\end{array}\right) \frac{1}{n_{2} ! \beta_{g_{2}}^{n_{2}} \times \Gamma\left(m_{k_{d_{1}}}\right) \beta_{k_{d_{1}}}^{m_{k_{d_{1}}}}}\left(\frac{v \delta_{2}^{\text {th }}}{a_{2}}\right)^{n_{3}}\left(\frac{\delta_{2}^{t h}}{a_{2} \rho_{r}}\right)^{n_{2}-n_{3}} \\
& \times\left(\frac{v \delta_{2}^{t h}}{a_{2} \beta_{g_{2}}}+\frac{1}{\beta_{k_{d_{1}}}}\right)^{-n_{3}-m_{k_{d_{1}}}} \Gamma\left(n_{3}+m_{k_{d_{1}}}, \mu\left(\frac{v \delta_{2}^{t h}}{a_{2} \beta_{g_{2}}}+\frac{1}{\beta_{k_{d_{1}}}}\right)\right)
\end{aligned}
$$

Plugging above values of (16) and (17) into (15), After the implementation of the calculation, we have the outage probability for detecting $x_{2}$ at $D_{2}$ in special case of imperfect SIC can be formulated in closed-form as:

$$
\begin{aligned}
& O P_{2}^{i j s C}=1-\exp \left(-\frac{\delta_{1}^{i h}}{\left(a_{1} \rho_{r}-a_{2} \rho_{r} \delta_{1}^{i n}\right) \beta_{s_{2}}}\right)_{n=0}^{m_{n}-1} \sum_{n_{1}=0}^{m_{m_{2}}-1} \frac{1}{n ! \beta_{\beta_{2}}^{n}}\left(1-\exp \left(-\frac{\mu}{\beta_{k_{d_{1}}}}\right) \frac{\mu^{n_{1}}}{n_{1} ! \beta_{k_{d_{1}}}^{n_{1}}}\right)\left(\frac{\delta_{1}^{t h}}{a_{1} \rho_{r}-a_{2} \rho_{r} \delta_{1}^{t h}}\right)^{n}
\end{aligned}
$$

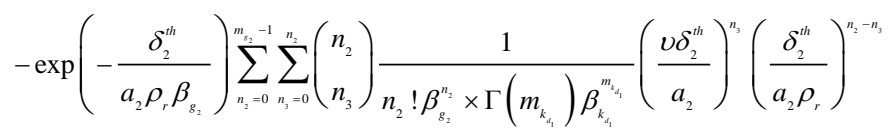

$$
\begin{aligned}
& \times\left(\frac{v \delta_{2}^{i n}}{a_{2} \beta_{g_{2}}}+\frac{1}{\beta_{k_{4}}}\right)^{-n_{3}-m_{k_{4}}} \Gamma\left(n_{3}+m_{k_{k_{4}}}, \mu\left(\frac{v \delta_{2}^{i n}}{a_{2} \beta_{g_{2}}}+\frac{1}{\beta_{k_{k_{4}}}}\right)\right)
\end{aligned}
$$

\subsection{The throughput on the outage probability}

Other metric provides further performance of such network. The throughput mainly depends on the outage probability. In particular, throughput performance can be obtained in three cases as follows:

$$
\begin{aligned}
& \mathrm{TH}_{1}=\left(1-O P_{1}\right) \times R, \\
& \mathrm{TH}_{2}^{p S I C}=\left(1-O P_{2}^{p S I C}\right) \times R, \\
& \mathrm{TH}_{2}^{i p S I C}=\left(1-O P_{2}^{i p S I C}\right) \times R .
\end{aligned}
$$

\section{SIMULATION RESULTS}

Novel multiple access for cooperative networks with nakagami-m fading: system... (Tu-Trinh Thi Nguyen) 
This section provides numerical results. It can be seen outage performance of two users versus transmit SNR at the BS as in Figure 2. At ipSIC case, user 2' performance will meet floor value at high SNR. The varying target rates contribute to outage performance and it shows that the better outage performance as lower target rates required. While Figure 3 illustrates throughput performance versus transmit SNR.

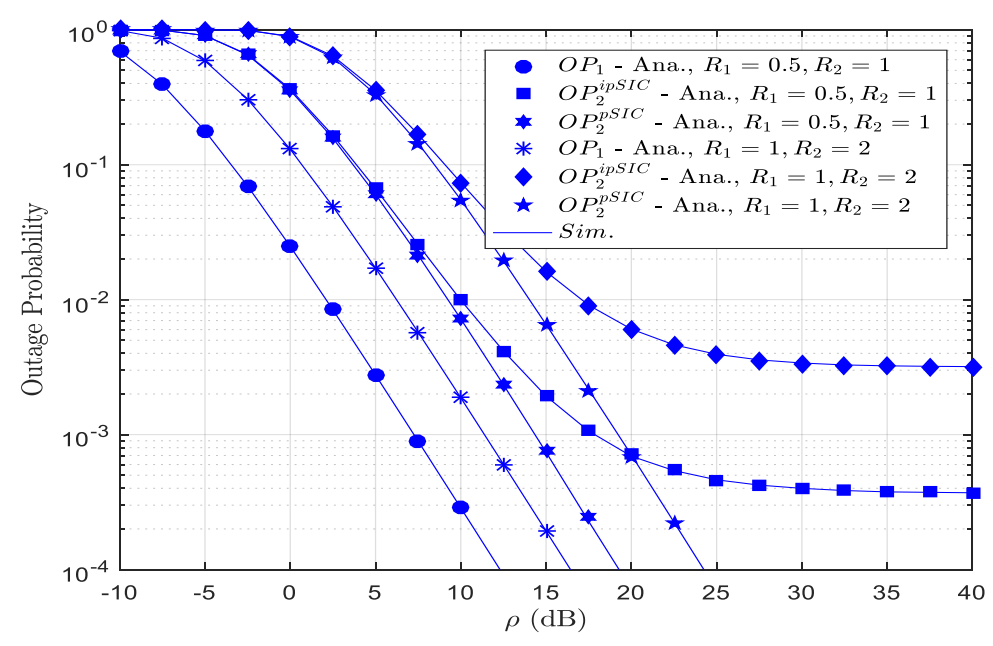

Figure 2. Outage probability vs. the transmit SNR with $a_{1}=0.9, a_{2}=0.1, m=2, \alpha=2, \kappa=0.005$,

$$
d_{1 r}=0.5, d_{r 1}=0.5, d_{r 2}=0.25
$$

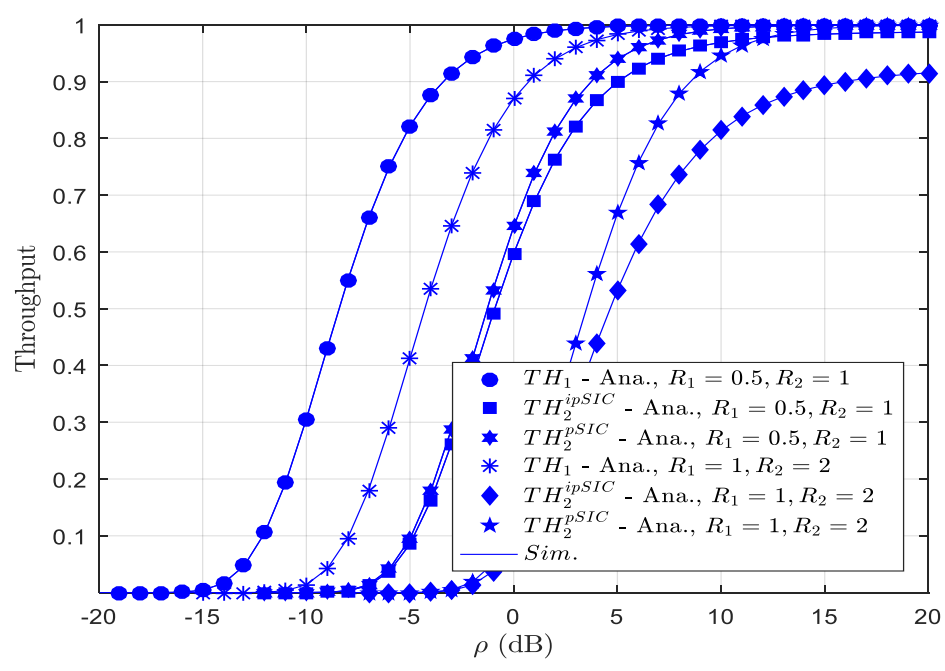

Figure 3. Throughput performance vs. the transmit SNR with $a_{1}=0.9, a_{2}=0.1, m=2, \alpha=2, \kappa=0.03$

$$
, d_{1 r}=0.5, d_{r 1}=0.5, d_{r 2}=0.25
$$

Figure 4 examines impact of Nakagami-m fading factors on outage performance as considering range of interference levels $\kappa=-50 \rightarrow 0(d B)$. It can be seen clearly that higher level of interference of SIC imperfection leads to outage condition. 


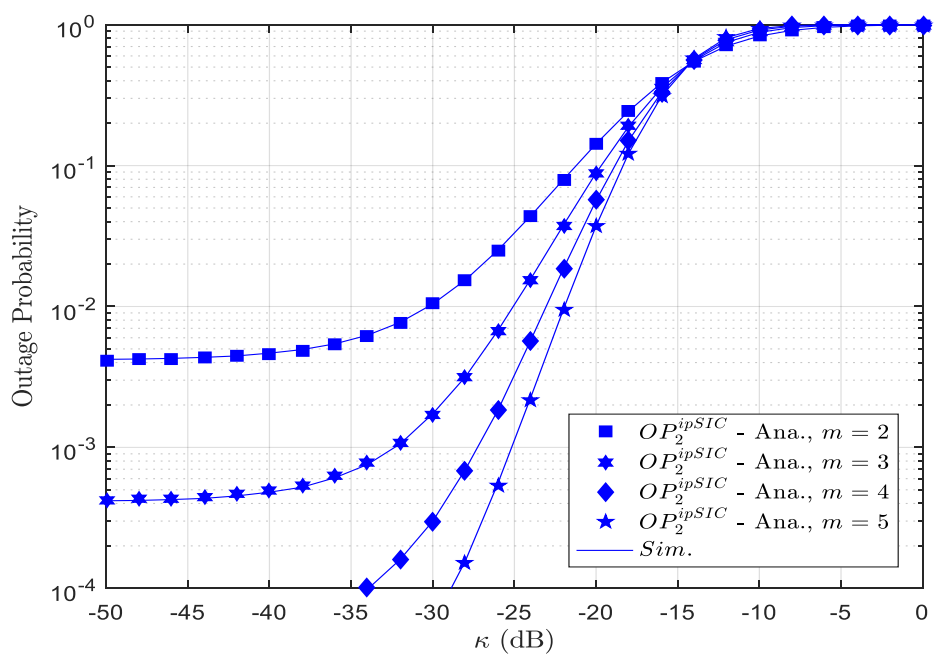

Figure 4. Outage probability under impact of $\kappa$ with $a_{1}=0.9, a_{2}=0.1, R_{1}=0.5, R_{2}=1$,

$$
\alpha=2, \kappa=0.03, d_{1 r}=0.5, d_{r 1}=0.5, d_{r 2}=0.25 \text {. }
$$

\section{CONCLUSION}

In this paper, the performance of the downlink NOMA system with two hop transmission over Nakagami-m fading channels is investigated. First, we derived expressions of outage probability to provide the analytical evaluation for each user. To confirm the accuracy of the analytical results, Monte-Carlo simulations are performed. In addition, by selecting suitable target rate coefficients, Nakagami-m fading factors, it can be achieved the outage behaviors of the users. Then, we evaluated the throughput of the NOMA system based upon the outage probability of each user. By conducting numerical research on several parameters, the lowest outage performance and highest throughputs were obtained.

\section{ACKNOWLEDGEMENT}

This research is funded by Foundation for Science and Technology Development of Ton Duc Thang University (FOSTECT), website: http://fostect.tdt.edu.vn, under Grant FOSTECT.2017.BR.21.

\section{REFERENCES}

[1] Q. Zhang, et al., "Buffer-aided non-orthogonal multiple access relaying systems in Rayleigh fading channels," IEEE Trans. Commun, vol. 65, no. 1, pp. 95-106, 2017.

[2] G. Cai, et al., "Design and analysis of relay-selection strategies for two-way relay network-coded DCSK systems," IEEE Trans. Veh. Technol, vol. 67, no. 2, pp. 1258-1271, 2018.

[3] M. F. Kader and S. Y. Shin, "Cooperative relaying using space-time block coded non-orthogonal multiple access," IEEE Trans. Veh. Technol, vol. 66, no. 7, pp. 5894-5903, 2017.

[4] Z. Yu, et al., "Non-orthogonal multiple access relaying with truncated ARQ," IET Commun., vol. 11, no. 4, pp. 514-521, 2017.

[5] X. X. Nguyen and D. T. Do, "Optimal power allocation and throughput performance of full-duplex DF relaying networks with wireless power transfer-aware channel," EURASIP Journal on Wireless Communications and Networking, p. 152, 2017.

[6] X. X. Nguyen and D. T. Do, "Maximum Harvested Energy Policy in Full-Duplex Relaying Networks with SWIPT," International Journal of Communication Systems (Wiley), vol. 30, no. 17, 2017.

[7] D. T. Do, "Energy-aware two-way relaying networks under imperfect hardware: optimal throughput design and analysis," Telecommunication Systems, vol. 62, no. 2, pp. 449-459, 2016.

[8] T. L. Nguyen and D. T. Do, "A new look at AF two-way relaying networks: energy harvesting architecture and impact of co-channel interference," Annals of Telecommunications, vol. 72, no. 11, pp. 669-678, 2017.

[9] D. T. Do, "Time Power Switching based Relaying Protocol in Energy Harvesting Mobile Node: Optimal Throughput Analysis," Mobile Information Systems, 2015.

[10] D. T. Do, "Power Switching Protocol for Two-way Relaying Network under Hardware Impairments," Radioengineering, vol. 24, no. 3, pp. 765-771, 2015.

[11] D. T. Do and H. S. Nguyen, "A Tractable Approach to Analyze the Energy-Aware Two-way Relaying Networks in Presence of Co-channel Interference," EURASIP Journal on Wireless Communications and Networking, p. 271, 2016.

\footnotetext{
Novel multiple access for cooperative networks with nakagami-m fading: system... (Tu-Trinh Thi Nguyen)
} 
[12] M. N. Pham, et al., "Energy Harvesting Assisted Cognitive Radio: Random Location-based Transceivers Scheme and Performance Analysis," Telecommunication Systems, vol. 67, no. 1, pp. 123-132, 2018.

[13] D. T. Do, et al., "Wireless Powered Relaying Networks under Imperfect Channel State Information: System Performance and Optimal Policy for Instantaneous Rate," RadioEngineering, vol. 26, no. 3, pp. 869-877, 2017.

[14] H. S. Nguyen, et al., "Exploiting Hybrid Time Switching-based and Power Splitting-based Relaying Protocol in Wireless Powered Communication Networks with Outdated Channel State Information, " Automatika - Journal for Control, Measurement, Electronics, Computing and Communications, vol. 58, no. 1, pp. 111-118, 2017.

[15] D. T. Do, "Optimal Energy Harvesting Scheme for Power Beacon-Assisted Wireless-Powered Networks," Indonesian Journal of Electrical Engineering and Computer Science, vol. 7, no. 3, pp. 802-808, 2017.

[16] D. A. Nguyen and D. T. Do, "The Maximal SINR Selection Mode for 5G Millimeter-Wave MIMO: Model Systems and Analysis," Indonesian Journal of Electrical Engineering and Computer Science, vol. 7, no. 1, 2017.

[17] D. T. Do, et al., "NOMA-Assisted Multiple Access Scheme for IoT Deployment: Relay Selection Model and Secrecy Performance Improvement," Sensors, vol. 19, no. 3, p. 736, 2019.

[18] D. T. Do and C. B. Le, "Application of NOMA in Wireless System with Wireless Power Transfer Scheme: Outage and Ergodic Capacity Performance Analysis,” Sensors, vol. 18, no. 10, p. 3501, 2018.

[19] T. L. Nguyen and D. T. Do, "Power Allocation Schemes for Wireless Powered NOMA Systems with Imperfect CSI: System model and performance analysis," International Journal of Communication Systems, vol. 31, no. 15, p. $3789,2018$.

[20] D. T. Do and M. S. Van Nguyen, "Device-to-device transmission modes in NOMA network with and without Wireless Power Transfer," Computer Communications, vol. 139, pp. 67-77, May 2019.

[21] D. T. Do, et al., "Wireless Powered Cooperative Relaying using NOMA with Imperfect CSI," in Proc. of IEEE Globecom Workshops (GC Wkshps), Abu Dhabi, UAE, pp. 1-6, 2018.

[22] D. T. Do, et al., "On Exact Outage and Throughput Performance of Cognitive Radio based Non-Orthogonal Multiple Access Networks With and Without D2D Link," Sensors, vol. 19, p. 3314, 2019.

[23] D. T. Do and A. T. Le, "NOMA based cognitive relaying: Transceiver hardware impairments, relay selection policies and outage performance comparison," Computer Communications, vol. 146, pp. 144-154, 2019.

[24] J. Men, et al., "Performance analysis for downlink relaying aided non-orthogonal multiple access networks with imperfect CSI over Nakagami-m fading," IEEE Access, vol. 5, pp. 998-1004, 2017. 